LAWRENCE LIVERMORE N A TION A L LABORATORY
Isochoric Heating of
Solid-Density Matter with an
Ultrafast Proton Beam

P. K. Patel, A. J. Mackinnon, M. H. Key, T. E. Cowan, M. E. Foord, M. Allen, D. F. Price, H. Ruhl, P. T. Springer, R. Stephens

January 9, 2004

Physical Review Letters 
This document was prepared as an account of work sponsored by an agency of the United States Government. Neither the United States Government nor the University of California nor any of their employees, makes any warranty, express or implied, or assumes any legal liability or responsibility for the accuracy, completeness, or usefulness of any information, apparatus, product, or process disclosed, or represents that its use would not infringe privately owned rights. Reference herein to any specific commercial product, process, or service by trade name, trademark, manufacturer, or otherwise, does not necessarily constitute or imply its endorsement, recommendation, or favoring by the United States Government or the University of California. The views and opinions of authors expressed herein do not necessarily state or reflect those of the United States Government or the University of California, and shall not be used for advertising or product endorsement purposes. 


\title{
Isochoric Heating of Solid Density Matter with an Ultrafast Proton Beam
}

\author{
P.K. Patel ${ }^{1}$, A.J. Mackinnon ${ }^{1}$, M.H. Key ${ }^{1}$, T.E. Cowan ${ }^{2}$, M.E. Foord ${ }^{1}$, \\ M. Allen ${ }^{1}$, D.F. Price ${ }^{1}$, H.Ruhl ${ }^{2}$, P.T. Springer ${ }^{1}$ and R. Stephens ${ }^{2}$ \\ ${ }^{1}$ Lawrence Livermore National Laboratory, Livermore, CA 94550, U.S.A. \\ ${ }^{2}$ General Atomics, P.O. Box 85608, San Diego, CA 92186, U.S.A.
}

\begin{abstract}
A new technique is described for the isochoric heating (i.e., heating at constant volume) of matter to high energy-density plasma states $\left(>10^{5} \mathrm{~J} / \mathrm{g}\right)$ on a picosecond timescale $\left(10^{-12}\right.$ sec). An intense, collimated, ultrashort-pulse beam of protons-generated by a highintensity laser pulse — is used to isochorically heat a solid density material to a temperature of several $\mathrm{eV}$. The duration of heating is shorter than the timescale for significant hydrodynamic expansion to occur, hence the material is heated to a solid density warm dense plasma state. Using spherically-shaped laser targets a focused proton beam is produced and used to heat a smaller volume to over $20 \mathrm{eV}$. The technique described of ultrafast proton heating provides a unique method for creating isochorically heated highenergy density plasma states.
\end{abstract}


Today's generation of ultrahigh-power lasers have the ability to compress and heat matter to energy densities similar to those at the centers of stars, giving them a leading role in the laboratory investigation of extreme states of matter, with major applications in planetary and stellar astrophysics [1] and fusion energy research [2]. Laboratory studies of plasmas enable measurements of fundamental material properties - such as the equation of state and opacity—needed to formulate and benchmark theoretical plasma models [3-6]. Ideally such measurements would be made on uniformly heated plasmas in a singledensity and single-temperature state. However, the production of plasmas in such idealised states is rather problematic because the heating or energy deposition is required to be both extremely rapid and uniform throughout the material — that is, isochoric heating, or heating at constant volume, is required. Established methods for volumetric heating such as laserdriven shock heating [7], x-ray heating [8,9], and ion heating [10] whilst relatively fast $\left(10^{-}\right.$ ${ }^{9}-10^{-6} \mathrm{sec}$ ) are still longer than the typical timescales over which significant hydrodynamic expansion can occur $\left(10^{-12}-10^{-11} \mathrm{sec}\right)$. Direct heating with intense sub-picosecond laser pulses $\left(10^{-12} \mathrm{sec}\right)$ is possible but results in highly non-uniform heating due to the laser absorption being localised within a skin depth $(<100 \mathrm{~nm})$ of material [11]. In this Letter we present a new approach to the heating of dense plasma states which overcomes both of these problems. This method uses an intense, collimated, laser-generated proton beam to volumetrically heat solid density material to warm dense states on a picosecond timescale.

The discovery that intense, highly directional proton beams could be generated during the interaction of an ultraintense laser pulse with a solid target was made relatively recently $[12,13]$. These and subsequent experiments characterising the proton beams have 
revealed a unique combination of properties including peak proton energies of $55 \mathrm{MeV}$, conversion efficiencies ranging between $2-7 \%$, a temporal duration of $<5 \mathrm{ps}$, and a narrow half-cone angle of emission of $15-20^{\circ}$ [13-15]. A high-intensity sub-picosecond laser pulse incident on a thin $\mathrm{Al}$ foil ponderamotively accelerates electrons from the interaction region into the target with relativistic energies. The electrons emerging at the rear surface induce a large electrostatic charge separation field, which in turn accelerates positive ions-mostly protons from a hydrocarbon contaminant layer-from the rear surface to multi-MeV energies, over a distance of a few microns. The protons are accelerated from the rear surface in a well-defined, highly directional beam normal to the target $[16,17]$. Simulations have shown that by curving the target rear surface the proton beam could potentially be focused to a far higher energy-density [17]. This Letter describes the application of an ultrashort-pulse proton beam to volumetrically heat a solid density material to a $4 \mathrm{eV}$ plasma state. The material, a $10 \mu \mathrm{m}$ thick $\mathrm{Al}$ foil, is isochorically heated by the protons at solid density on a few picoseconds timescale—a time over which negligible hydrodynamic expansion of the plasma occurs. In addition, a technique is demonstrated for focusing the proton beam to even higher flux densities. This technique leads to the heating of a smaller volume of solid material to over $20 \mathrm{eV}$ in temperature.

The experiments were performed on the 100 TW JanUSP laser at Lawrence Livermore National Laboratory. JanUSP is a Titanium Sapphire (Ti:S) laser operating at a wavelength of $800 \mathrm{~nm}$ and delivering $10 \mathrm{~J}$ of energy in a $100 \mathrm{fs}$ duration pulse [18]. The laser is focused by an $\mathrm{f} / 2$ off-axis parabola to a $5 \mu \mathrm{m}$ FWHM (full width at half maximum) spot. For these experiments the laser spot was defocused at the target plane to a $50 \mu \mathrm{m}$ 
diameter with an average intensity of $5 \times 10^{18} \mathrm{Wcm}^{-2}$ in order to optimize the proton beam for this application. The proton beam was characterized using a stack of 20 sheets of radiochromic film (RCF) placed $25 \mathrm{~mm}$ behind a $20 \mu \mathrm{m}$ thick $\mathrm{Al}$ foil target. $\mathrm{RCF}$ is an absolutely calibrated dosimetry film measuring total radiation dose or deposited energy. The recorded images show the angular pattern of the beam in the narrow proton energy band depositing energy in each sheet of film. By structuring the rear surface of the $\mathrm{Al}$ foil an intensity variation was imprinted on the proton beam [19] which provided a measurement of the size of the emitting region on the foil. The source diameter ranged from 250 to $80 \mu \mathrm{m}$ for the recorded range of proton energies from 4 to $12 \mathrm{MeV}$, and was much larger than the laser focal spot. This appears consistent with reflux spreading of the electrons within the target [15]. For the subsequent heating and focusing parts of the experiment a $10 \mu \mathrm{m}$ thick $\mathrm{Al}$ foil with a smooth rear surface was used to generate the proton beam. The energy spectrum of the protons, measured with RCF, was close to an exponential with a temperature of $1.5 \pm 0.2 \mathrm{MeV}$ and a total energy of $0.1-0.2 \mathrm{~J}$, or $1-2 \%$ of the incident laser energy.

Figure 1a shows the experimental setup and target geometries. A planar case was studied first in which the proton beam is produced from a $10 \mu \mathrm{m}$ planar Al foil, and a second $10 \mu \mathrm{m} \mathrm{Al}$ foil is placed behind the first at a distance of $250 \mu \mathrm{m}$. In the focusing case the proton beam is produced from a $10 \mu \mathrm{m}$ thick, $320 \mu \mathrm{m}$ diameter hemispherical $\mathrm{Al}$ shell, and a second $10 \mu \mathrm{m} \mathrm{Al}$ foil is placed in a plane coinciding with the geometric center of the shell. The temperature of the proton heated foil was determined with a fast optical streak camera recording Planckian thermal emission from the hot rear surface. An absolute 
single wavelength measurement was made using a $570 \mathrm{~nm}$ interference filter. The overall temporal and spatial resolution was $70 \mathrm{ps}$, and $5 \mu \mathrm{m}$, respectively. The $10 \mu \mathrm{m}$ thick proton heated foil blocked any direct light from the primary laser-irradiated target.

The streak camera data obtained for the two target geometries, each with $10 \mathrm{~J}$ of laser energy incident on target, are shown in Fig. 1b. For the planar foil case (left image) we observe quite uniform emission from a large area of the secondary foil $(186 \mu \mathrm{m}$ FWHM). The onset of the emission is rapid-shorter than the time resolution of the streak camera — and decreases slowly over the following 800 ps. This temporal behavior (a rapid rise with a slow fall-off) is consistent with that from a body which is heated isochorically to some temperature and which then under its own pressure expands and cools. The spatial extent of $186 \mu \mathrm{m}$ is in good agreement with our measurement of the maximum proton source size of approximately $250 \mu \mathrm{m}$ at the lowest recorded proton energy (NB. the protons primarily responsible for the heating at a depth of $10 \mu \mathrm{m}$ have energies in a band around $0.9 \mathrm{MeV}$ ). With the hemispherical foil (right image) we observe a dramatic reduction in the size of the heated region (46 $\mu \mathrm{m}$ FWHM) coupled with a marked increase in the emission intensity (approximately a factor of 8). The factor of 4 reduction in the spatial extent in one dimension corresponds to a 16 times smaller heated area.

An interferometer was used to simultaneously monitor the foils for signs of plasma formation. The interferometry beam was a frequency-doubled 100 fs pulse directed along the target surface and timed to arrive 180 ps after the main pulse. Figure 2 shows the interferogram for the $320 \mu \mathrm{m}$ hemispherical shell target corresponding to the same shot shown in Fig. 1b. The large fringe shifts on the left of the target arise from the blow-off 
plasma covering the outer surface of the hemispherical shell (the laser is incident from the left). The right side of the image corresponds to the rear surface of the secondary foil. A small region of expanding plasma is clearly visible. The plasma, originating from the rear surface, is centred along the central axis of the hemisphere, and extends laterally over approximately $50 \mu \mathrm{m}$, in good agreement with the $46 \mu \mathrm{m}$ heated region measured with the streak camera. Taken together these observations provide a strong indication of the ballistic focusing of the proton beam, and of the corresponding enhancement in its flux density.

An absolute single wavelength intensity measurement of the rear surface emission enables us to estimate the rear side temperature of the proton heated foil. Absolute calibration of the streak camera and transmission optics in the beam path provided an overall accuracy of $\pm 25 \%$. The radiation-hydrocode LASNEX [20] was used to model the hydrodynamic expansion and optical emission of the foil, assuming it to be instantaneously heated to some initial temperature. The simulated emission at $570 \mathrm{~nm}$ from the rear surface was then compared with the absolute intensity measurements.

Taking lineouts from the two images in Fig. $1 \mathrm{~b}$ we obtain peak emission values of $5.7 \times 10^{14}$ and $4.3 \times 10^{15}$ ergs $\mathrm{s}^{-1} \mathrm{~cm}^{-2} \mathrm{keV}^{-1}$ respectively. Fitting to these peak values LASNEX modeling indicates for the planar heating case an initial temperature of the $\mathrm{Al}$ foil of $4 \pm 1 \mathrm{eV}$, and for the focused heating case a temperature of $23 \pm 6 \mathrm{eV}$. The comparison between experiment and simulation for this latter case is shown in Fig. 3. We note that the fall-off in the emission intensity over the first 400 ps matches the data very closely. Since both the peak intensity and the fall-off are strong functions of the plasma temperature, this 
good agreement gives us an added degree of confidence in the accuracy of the temperature measurement. The rise in signal at 400 ps may be due to gradient effects from the front of the foil such as a shockwave reaching the rear surface.

The proton beam flux required to heat the foils to the observed temperatures was estimated using a Monte Carlo simulation [21]. Protons with an exponential energy spectrum of $1.5 \mathrm{MeV} \mathrm{kT}$ were injected into a $10 \mu \mathrm{m}$ thick Al foil. Energy loss and energy deposition as a function of distance were computed. The energy deposition at a depth of 10 $\mu \mathrm{m}$ was found to be $9.2 \times 10^{-7} \mathrm{~J} / \mathrm{g}$ per incident proton. Comparing to the evaluated energy density of $7.3 \times 10^{5} \mathrm{~J} / \mathrm{g}$ at $23 \mathrm{eV}$ [3] requires a total of $7.9 \times 10^{11}$ protons focused to the observed $46 \mu \mathrm{m}$ diameter spot. The total energy in such a distribution is $190 \mathrm{~mJ}$, or $1.8 \%$ of the incident laser energy. Although approximate, this figure is entirely consistent with our previous estimates of a $1-2 \%$ conversion efficiency to protons, showing that there is sufficient energy in the focused proton beam to induce isochoric heating to the level observed.

The focusing in a purely ballistic limit can be estimated by considering the flow angle deviation with source radius, as seen from a planar foil, and applying it to the hemispherical shell. The real behaviour is expected to deviate from a pure ballistic case because of the spatio-temporal varying electron density and accelerating sheath field at the target rear surface. To gain insight into the complex focusing dynamics we carried out 2-D particle-in-cell (PIC) simulations which model the laser absorption, electron generation and propagation, and proton acceleration. A spatio-temporal gaussian pulse with $50 \mu \mathrm{m}$ and $100 \mathrm{fs}$ FWHM, respectively, is incident at the left boundary of a $200 \times 200 \mu \mathrm{m}$ 
simulation box. The resulting peak laser intensity is $5 \times 10^{18} \mathrm{Wcm}^{-2}$. The target consists of a $10 \mu \mathrm{m}$ thick, $125 \mu \mathrm{m}$ radius $\mathrm{Al}$ shell with a $10 \mu \mathrm{m}$ thick flat $\mathrm{Al}$ foil positioned at the centre of curvature of the shell. A $0.1 \mu \mathrm{m} \mathrm{H}$ layer is added to the inner surface of the hemisphere to simulate the proton-producing hydrocarbon layer. Figure 4 shows a result from the simulation, an electric field density map at a time 3.4 ps after the peak of the laser pulse. At this time the leading edge of the ion front has almost reached the rear foil. The accelerating sheath field can be seen to cover a large area of the inner surface of the hemisphere, producing a substantial degree of directed proton acceleration.

In conclusion, we have shown that an ultrashort-pulse beam of energetic protons, generated with a high-intensity laser pulse, is capable of isochorically heating a material to a warm dense plasma state at several $\mathrm{eV}$. The protons volumetrically heat a $10 \mu \mathrm{m}$ thick $\mathrm{Al}$ foil over an area of almost $200 \mu \mathrm{m}$ in diameter. Using hemispherically-shaped targets we have been able to generate a focused proton beam with a corresponding enhancement in flux of almost an order of magnitude. The focused beam enabled heating of a localised 50 $\mu \mathrm{m}$ diameter area to $23 \mathrm{eV}$. We note that the $23 \mathrm{eV}, 7.3 \times 10^{5} \mathrm{~J} / \mathrm{g}$ solid density plasma reported herein was produced with a $10 \mathrm{~J}$ laser generating a $0.2 \mathrm{~J}$ proton beam; however, the world's largest sub-picosecond lasers are capable of delivering laser energies of $500 \mathrm{~J}$, and generating proton beams with up to $30 \mathrm{~J}$, or 150 times the proton energy produced here $[13,22]$. Applying the techniques of proton heating and focusing at such facilities could enable isochoric heating of solid density plasmas to $\mathrm{keV}$ temperatures and gigabar pressures. This would open up new opportunities and directions in high energy-density physics and fusion energy research. 
This work was funded under the auspices of the U.S. Department of Energy by UC LLNL under Contract No. W-7405-ENG-48. 


\section{References}

1. Remington, B.A., Arnet, D., Drake, R.P. \& Takabe, H. Science 284, 1488-1493 (1999).

2. Nuckolls, J., Wood, L., Thiessen, A. \&Zimmerman, G. Nature 239, 139-142 (1972).

3. More, R.M., Warren, K.H., Young, D.A. and Zimmerman, G.B. Phys. Fluids, 31, 3059-3078 (1988).

4. Holian, K.S. LANL Report No. LA-10160-MS UC-34 (1984).

5. Collins, G.W. et al. Science 281, 1178 (1998)

6. Rogers, F.J. and Iglesias, C.A. Astrophysical Opacity. Science 263, 50-55 (1994).

7. Da Silva L.B. et al. Phys. Rev. Lett. 78, 483 (1997).

8. Perry, T.S. et al. Phys. Rev. E 54, 5617-5631 (1996)

9. MacFarlane, J.J. et al. Phys. Rev. E 66, 046416 (2002).

10. Hoffmann, D.H.H. et al. Phys. Plasmas 9, 3651-3654 (2002).

11. Audebert, P. et al. Phys. Rev. Lett. 89, 265001 (2002).

12. Clark, E. et al. Phys. Rev. Lett. 84, 670-673 (2000).

13. Snavely, R. et al. Phys. Rev. Lett. 85, 2945-2948 (2000).

14. Borghesi, M. et al. Phys. Plasmas 9, 2214 (2002).

15. Mackinnon, A.J. et al. Phys. Rev. Lett. 88, 215006 (2002).

16. Hatchett. S.P. et al. Phys. Plasmas 7, 2076-2082 (2000).

17. Wilks, S.C. et al. Phys. Plasmas 8, 542-549 (2001).

18. Bonlie, J.D., Patterson, F., Price, D.F., White, B. and Springer, P.T. Appl. Phys. B 70, S155-S160 (2000). 
19. Cowan, T.E. and Ruhl, H., (submitted).

20. Zimmerman, G.B. and Kruer, W.L. Comments Plasma Phys. Control. Fusion 2, 51 (1975).

21. Ziegler, J.F., Biersack, J.P. \& Littmark, U. The Stopping and Range of Ions in Solids. Pergamon Press, New York (1996).

22. Perry, M.D. et al. Opt. Lett. 24, 160-162 (1999). 


\section{Figures}

Figure 1 (a) Experimental setup for flat and focusing target geometries. Each target consists of a flat or hemispherical $10 \mu \mathrm{m}$ thick Al target irradiated by the laser, and a flat 10 $\mu \mathrm{m}$ thick Al foil to be heated by the protons. (b) Corresponding streak camera images showing space- and time-resolved thermal emission at $570 \mathrm{~nm}$ from the rear side of the proton-heated foil. The streak camera images an $800 \mu \mathrm{m}$ spatial region with a $1 \mathrm{~ns}$ temporal window.

Figure 2 Interferogram of focusing target shot taken $180 \mathrm{ps}$ after incidence of the main pulse. The enlarged image on the right shows an approximately $50 \mu \mathrm{m}$ region of expanding plasma originating from the rear surface of the proton heated foil.

Figure 3 Comparison of time-dependent experimental (blue) and simulated (red) emission intensities for hemispherical target shot. The experimental curve is a temporal lineout spatially-integrated over the $46 \mu \mathrm{m}$ FWHM of the signal. The simulated curve is a LASNEX calculation of the $570 \mathrm{~nm}$ emission from a $23 \mathrm{eV}$ solid density Al plasma.

Figure 4 Particle-in-cell (PIC) calculation of the electric field density at 3.4 ps for a $5 \times 10^{18}$ $\mathrm{Wcm}^{-2}$ intensity laser pulse incident on a $250 \mu \mathrm{m}$ diameter, $10 \mu \mathrm{m}$ thick hemispherical $\mathrm{Al}$ shell. 
(a)

(b)

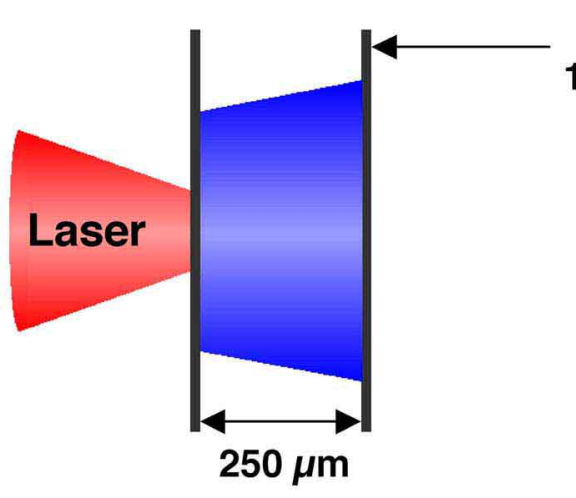

Proton-heated
$0 \mu \mathrm{m}$ thick Al foils
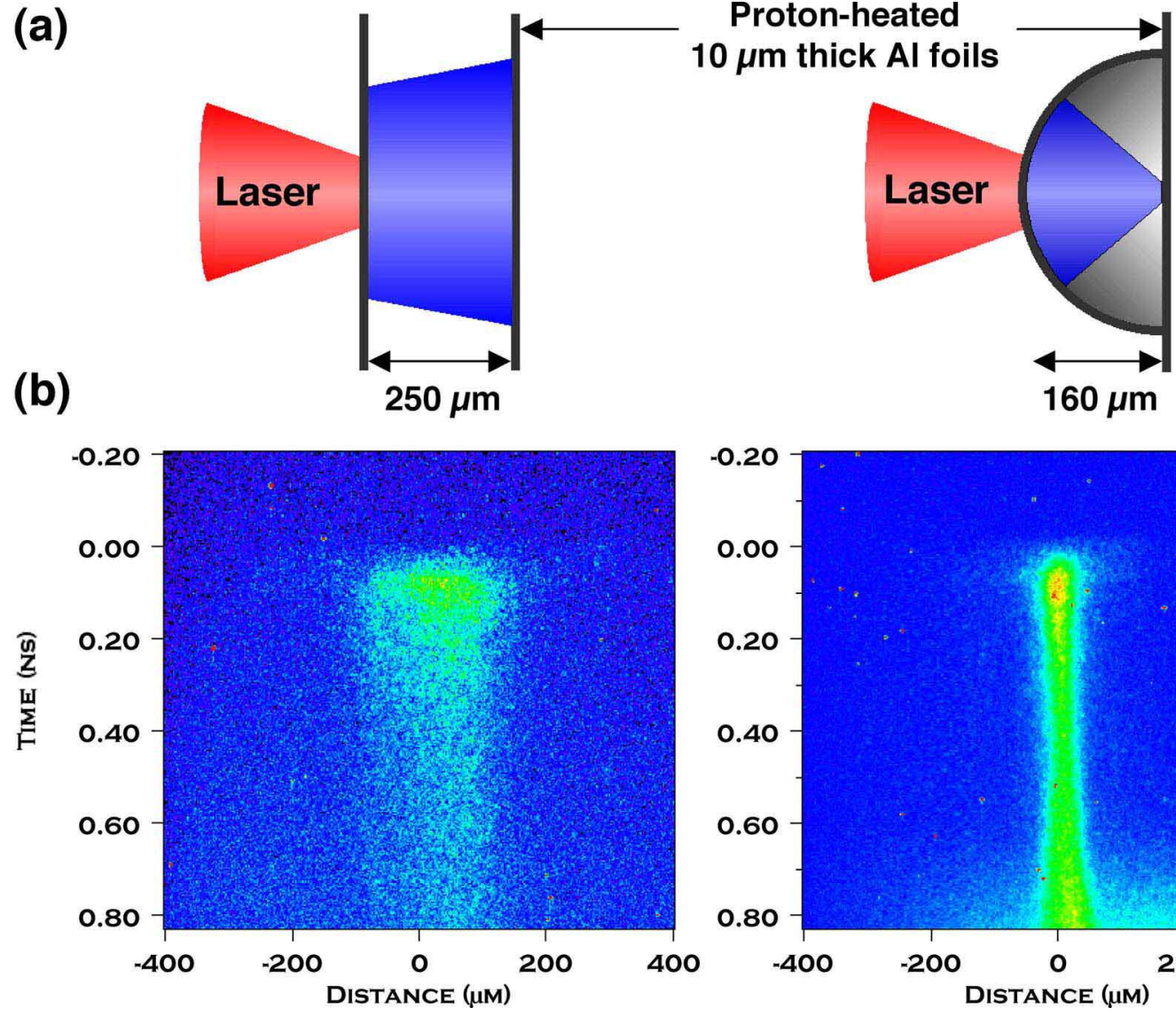

$160 \mu \mathrm{m}$

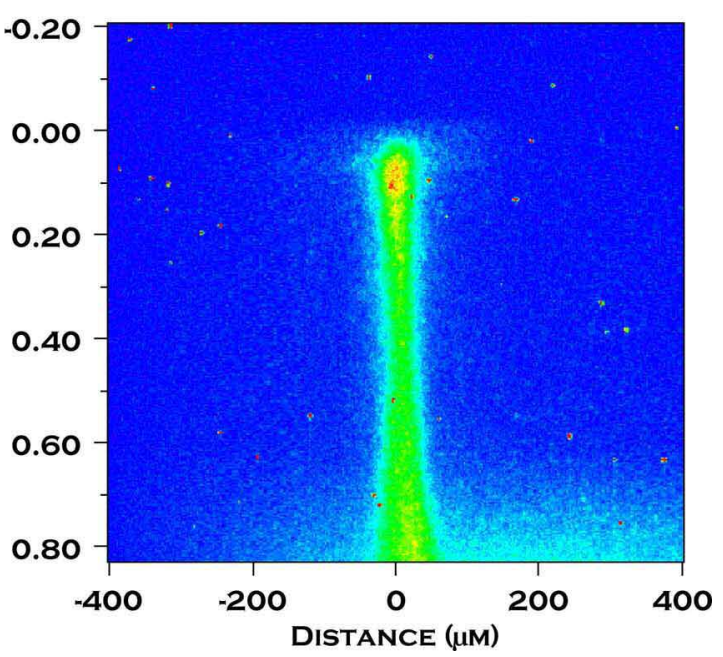

PK Patel et al., PRL, Fig. 1 

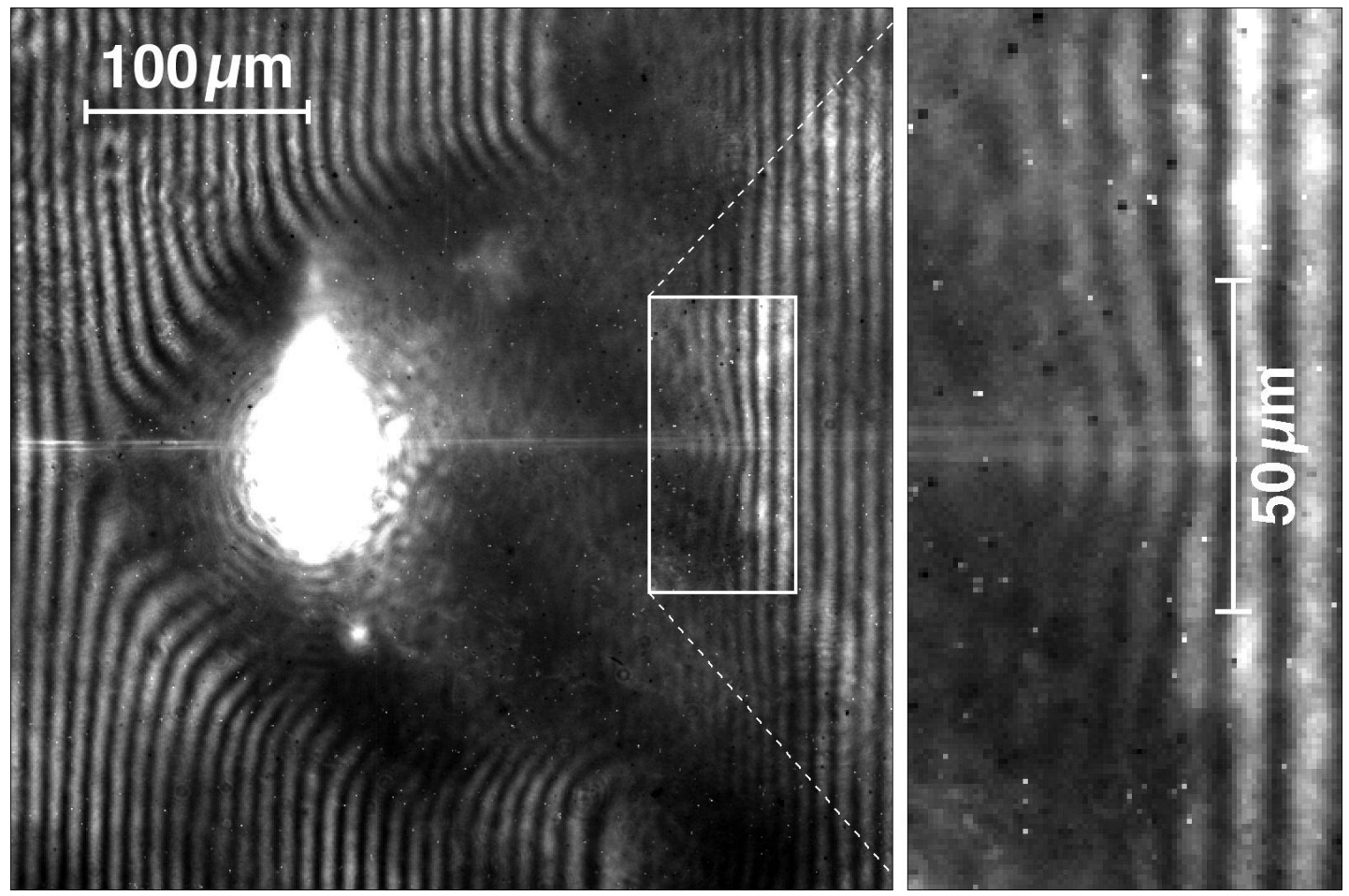

PK Patel et al., PRL, Fig. 2 


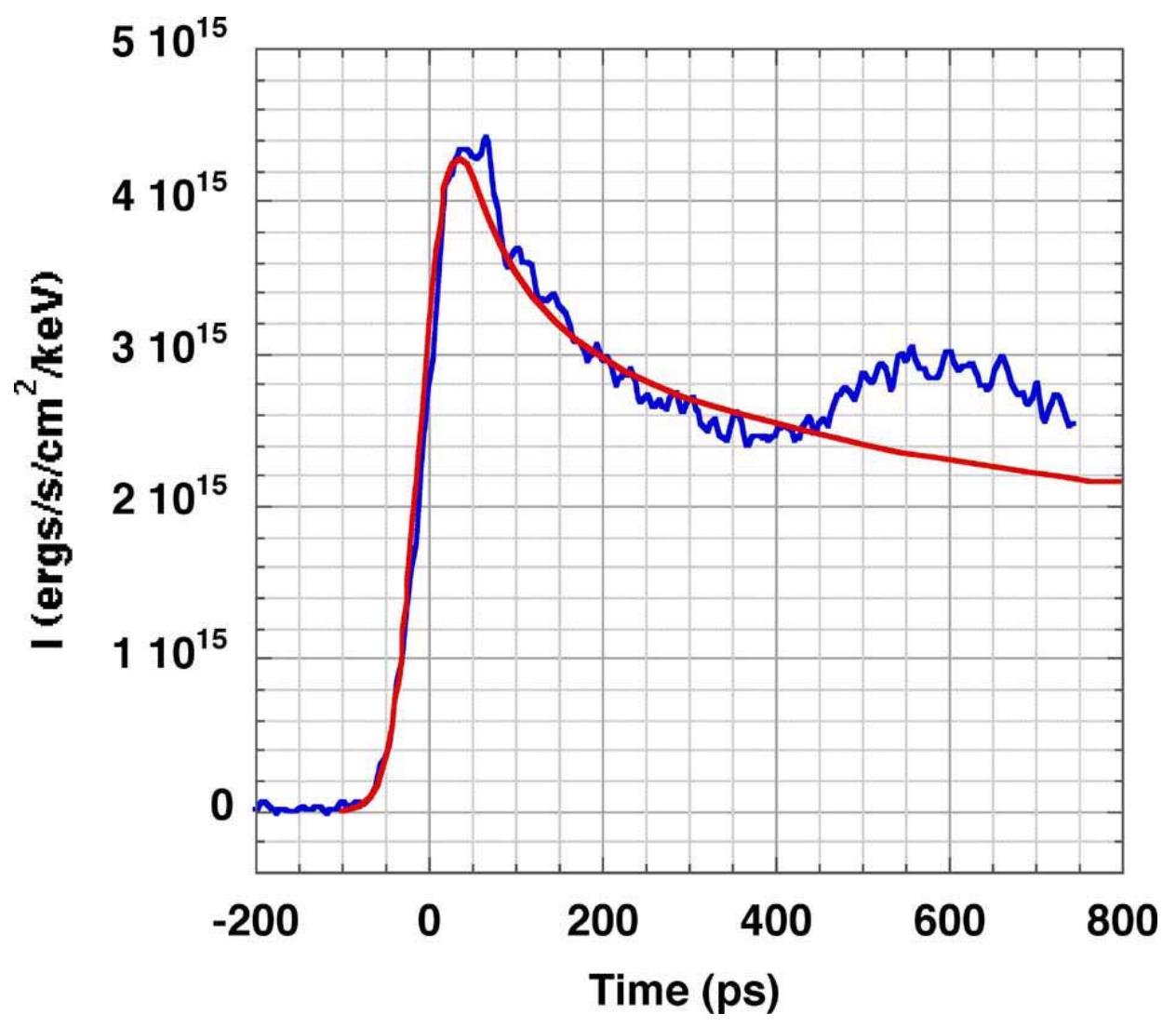

PK Patel et al., PRL, Fig. 3 


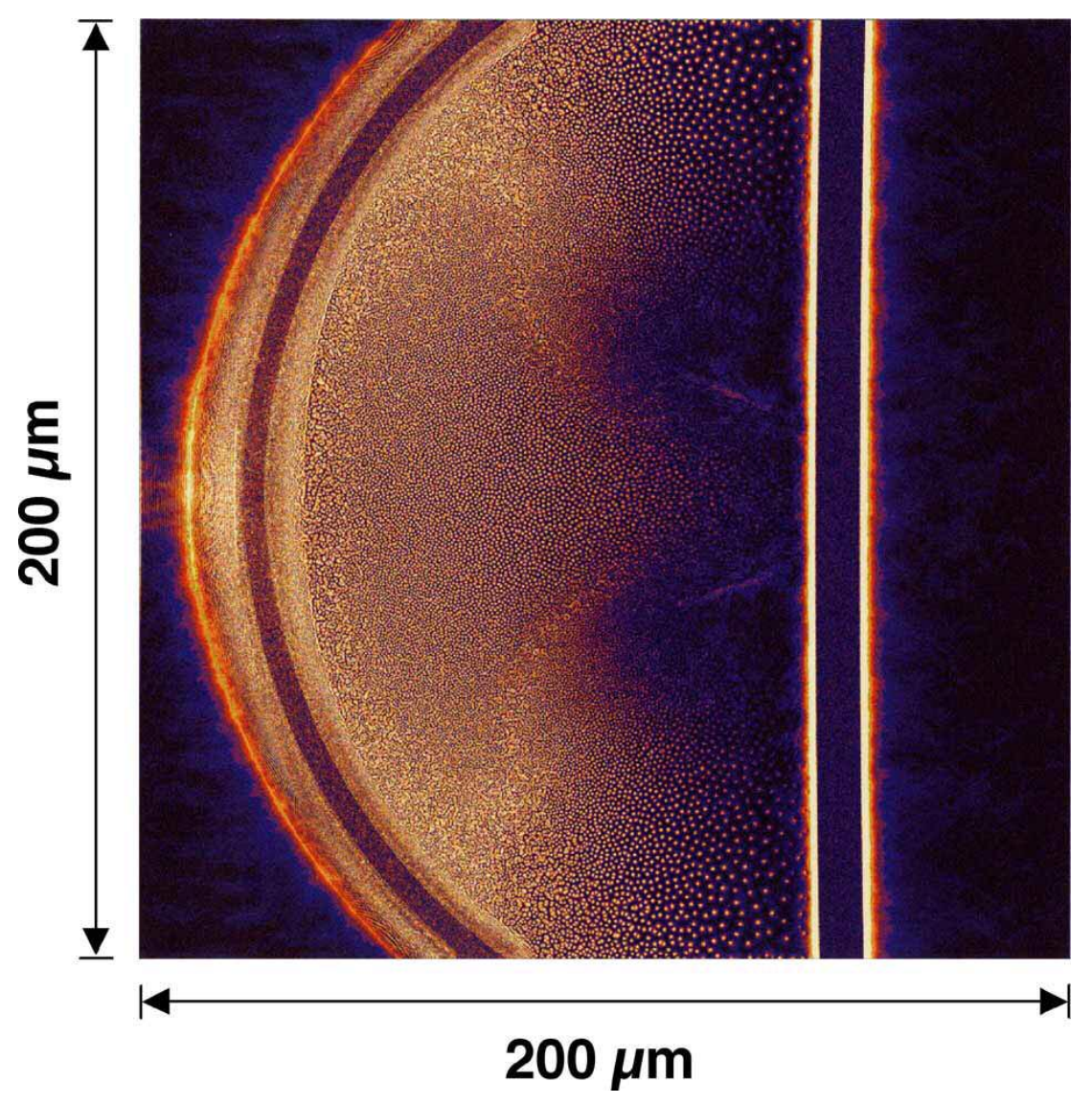

PK Patel et al., PRL, Fig. 4 